\title{
Alain Cuny, personnage claudélien
}

\author{
Bernard Hue
}

Sans cesse, Claudel me renvoie à moi-même ${ }^{1}$.

A. Cuny

$\mathrm{I}_{1}$ peut sembler paradoxal de voir dans le comédien qui fut l'ami d'Artaud', s'enthousiasma pour le surréalisme vers lequel l'orienta Reverdy, qui ne cessa d'accorder le plus grand intérêt à la psychanalyse dont René Laforgue et Françoise Dolto lui révélèrent l'efficacité, dans ce passionné d'astrologie, d'occultisme, chez cet homme tourmenté qui a pris ses distances avec le catholicisme dans lequel il fut élevé, un personnage qui serait sinon le double de Claudel, du moins l'image même du personnage type qui se dégage de l'ensemble du théâtre de l'auteur du Soulier de satin. Malgré de sensibles dissemblances entre le dramaturge et Alain Cuny, celuici, considéré comme la voix même de Claudel, reconnu comme le meilleur interprète de ces héros tragiques qui ont nom Simon Agnel, Couvre, Pierre de Craon, intrigue par plus d'un côté, en particulier par l'amitié que lui a vouée le poète et, plus encore par la manière dont il a perçu cet extraordinaire créateur qu'il assure avoir compris grâce à une parenté d'ordre existentiel, surprenante pour peu qu'on ignore le drame permanent qu'a été la vie de cet homme qui rêvait de devenir peintre, sans vocation pour le théâtre, qui, n'accordant au cinéma que peu d'intérêt acceptera pourtant d'être le réalisateur de L'Annonce faite à Marie.

C'est d'ailleurs cet interprète qui invite à voir en lui, non seulement sur scène, mais dans sa vie en général, un véritable personnage claudélien. Sa démarche, ses confidences, ses révélations parfois douloureuses au point de confiner au tragique, contribuent à éclairer l'étrange parenté que le grand tragédien s'est trouvée avec Claudel, en n'hésitant pas s'identifier à Cœuvre, Mesa, 


\section{Bernard Hue}

Rodrigue, à tous ces héros dont l'auteur s'est plu à préciser qu'ils constituaient son multiple habitant intérieur ${ }^{3}$.

Alain Cuny entre en scène dans l'œuvre et dans la vie de Claudel en 1941, à une époque où la censure vichyssoise le repère dans une pièce de Giono qui va se jouer aux Noctambules et le taxe, dans « un rapport accablant », d' « abominable acteur », permettant de présager un désastre inévitable ${ }^{4}$. Peu après, Claudel, comme le fit naguère Charles Dullin, découvre en Alain Cuny un remarquable acteur ${ }^{5}$.

A trente-trois ans, Cuny est presque un inconnu. Il a été simple figurant, en 1939, dans Madame sans gêne à la ComédieFrançaise ${ }^{6}$, et Carné ne tournera Les Visiteurs du soir qu'en 1942. Dans l'intervalle, vu son succès dans Le Bout de la route, il est invité au théâtre de l'CEuvre à incarner l'un des rôles les plus délicats de l'Annonce faite à Marie, celui de Pierre de Craon, personnage du $\mathrm{XV}^{\mathrm{e}}$ siècle, auquel le poète a fait subir d'importantes modifications depuis sa première apparition, dans la seconde version de La Jeune fille Violaine (1898-99), énigmatique personnage appelé à connaître encore d'autres modifications jusqu'à la création de la pièce à la Comédie-Française, six jours avant la mort de Claudel (février 1955). Si Cuny n'apparaît pas dans la distribution de cette ultime création du vivant de Claudel, c'est à lui que s'était adressé Hébertot quelques années plus tôt pour lui confier le rôle de Pierre de Craon, dans la version de 1948, dite définitive (mais remaniée largement dès 1938). Sa présence est désormais limitée à la durée du prologue, confrontation entre lui et Violaine. Entre ces deux contributions consacrées au même personnage de L'Annonce faite à Marie, Cuny est aussi apparu, aux côtés de Jean Vilar, dans le rôle de l'Ange Azarias de L'Histoire de Tobie et de Sara, lors du premier festival d'Avignon (1947).

Sa carrière dramatique ne se résume pas à ces interventions dans le théâtre de Claudel. Il est également remarqué dans des œuvres de Jean Anouilh (Eurydice), Skakespeare (Macbeth), Racine (Andromaque), Jean-Paul Sartre (Morts sans sépulture), Eugène O'Neil (Le deuil sied à Electre), etc. 
Après 1955, on le retrouve à Avignon et au théâtre de Chaillot où il est Cœuvre, le poète de La Ville. En 1959, l'Odéon Théâtre de France lui confie le rôle de Simon Agnel dans la création de Tête d'or, pièce de jeunesse dont Claudel a refusé toute création de son vivant, dans laquelle, aux côtés de Terzieff, Alain Cuny révèle à un large public que L'Annonce faite à Marie ne représente pas, il s'en faut, tout le théâtre de Claudel. Déjà, en 1943, Jean-Louis Barrault avait largement contribué à cette révélation en portant à la scène une version abrégée du Soulier de satin, suivie, en 1948, d'un Partage de midi revu par un auteur aussi soucieux, maintenant, de moralité que de dramaturgie. Dernière apparition scénique dans une pièce de Claudel, Cuny reprendra le rôle de Simon Agnel, en 1968, au Théâtre de France. C'est donc au total quatre héros claudéliens que Cuny aura incarnés : Pierre de Craon, l'Ange Azarias, Simon Agnel et Couvre? A mi-chemin entre le théâtre et la cérémonie religieuse, Cuny devait aussi avoir un rôle important dans Le Chemin de la Croix en donnant de ce poème une lecture à une voix dans la cathédrale de sa ville natale ${ }^{8}$.

A cette expérience multiple devait s'ajouter la participation du comédien à deux réalisations cinématographiques : L'Annonce faite à Marie, dont la réalisation lui a été demandée dès $1968^{\circ}$, où, faisant une entorse à la règle qu'il s'était imposée de ne pas employer de vedettes (la notion de star lui répugnant), il tiendrait lui-même le rôle d'Anne Vercors ${ }^{10}$; puis Camille Claudel, film de Bruno Nuytten (1988), où il interprète celui du père de Camille et Paul. Alors qu'il n'est jamais allé au-devant d'aucun engagement, qu'il n'a jamais sollicité explicitement tel ou tel rôle, ni fait valoir son éventuelle préférence pour tel au tel auteur, il est patent que Claudel, à plus d'un titre, a dominé sa carrière ${ }^{11}$.

Rien d'étonnant donc dans la déclaration lapidaire par laquelle, brossant son autoportrait, le comédien, plein d'assurance, est amené à se présenter :

Pierre de Craon, c'est moi ${ }^{12}$. 


\section{Bernard Hue}

Mais que veut-il faire entendre par là? Que signifie cette identification à l'emporte pièce avec un personnage dont on peut se demander si Cuny se réfère à lui comme à un type (impliquant l'existence d'un modèle précis, spécifiquement claudélien)? Le comédien dissipe l'ambiguité que peut faire naître la formulation de sa curieuse présentation de lui-même, car il lui arrive de recourir à d'autres personnages - qu'il a joués sur scène ou non - pour établir une étroite parenté, allant jusqu'à l'assimilation, entre lui et certains des principaux héros sortis de l'imagination de Claudel.

Je n'ai pas joué tous les personnages de Claudel, loin de là, mais tous ceux que j'ai joués ne font qu'un. Craon, c'est Tête d'or, Craon et Tête d'or, c'est Mesa ${ }^{13}$.

\section{Le héros claudélien vu par Cuny}

Le type même du personnage claudélien est donc, par excellence, aux yeux de Cuny, le héros malheureux de la pièce la plus autobiographique de Claudel. Si Simon Agnel est né de l'imagination du poète, ainsi que Craon introduit dans la seconde version de La jeune Fille Violaine, on doit souligner que Mesa se situe entre les œuvres de jeunesse (non historiques) et L'Annonce faite à Marie (version de 1911), proche de la Trilogie. En établissant l'équation «Craon et Tête d'or, c'est Mesa », Cuny suggère donc, compte tenu du caractère autobiographique de Partage de midi, qu'on peut voir en lui le double de Claudel, ce qui appelle quelques éclaircissements. Car comment comprendre cette surprenante déclaration, apparemment pleine de superbe :

On ne sait pas voir Claudel. [...] personne ne sait qui est Claudel! moi seul ${ }^{14} \ldots$

Le comédien ne suggère-t-il pas qu'il constitue une exception, que, grâce à son expérience personnelle, non limitée à la scène, il a l'avantage d'avoir eu une approche unique du poète, assimilable à une 


\section{Alain Cuny, personnage claudélien}

parenté, à une affinité d'ordre existentiel qui embrasse le domaine de la pensée, de la foi, du désir, tant celui du monde terrestre que celui de l'infini?

Alain Cuny qui, comme Claudel, se méfie des intellectuels, perçoit essentiellement une ressemblance morale, peut-être plus philosophique que spirituelle, entre le poète chrétien et lui, le rejeté, l'exclu, le maudit d'une famille très catholique. Et nul ne parle mieux que lui du personnage claudélien, qu'il a découvert en lui-même au point de se présenter tout naturellement comme Mesa, Pierre de Craon, Tête d'or. Nul, peut-être, n'aura mieux compris la parenté entre Claudel et le type même du héros claudélien :

Il est tronqué. Il est châtré. Il hurle dès qu'il se trouve en présence d'une femme. Et il use de tous les subterfuges. Sa sacralisation, son côté curé raté, en est un. C'est atroce. Dieu est absent. Mesa fait partie de la troupe. Peut-être pas Claudel lui-même. Lui avait une énergie de Titan. Il avait été trié parmi des milliards d'êtres. Il s'en est sorti. Il est parvenu à vivre cahin-caha, hérö̈quement. Il s'est donné une discipline draconienne et il s'y est plié comme un convers ${ }^{15}$.

Sans négliger le problème du théâtre, fondamental pour Claudel, secondaire pour lui, Cuny élargit sa réflexion, prenant en compte l'écriture à laquelle il accorde une très grande importance mais aussi l'homme qui écrit, situé dans des conditions particulières, appelé, contraint, jouet de forces secrètes antagonistes, déchirantes ou bénéfiques, toutes, en définitive, participant à une entreprise constructive. A ses yeux, Pierre de Craon est un appelé déchiré par deux forces antagonistes, par deux figures de l'absolu apparemment inconciliables, celle de la femme et celle de Dieu. Avatar de Claudel, Pierre de Craon, l'homme des ténèbres (où il guette Violaine), est aussi une sorte d'ange dans la lumière où il s'élève et où s'achève son œuvre d'artiste solitaire. Cuny voit en Claudel un chrétien luttant de toute son énergie contre le désespoir, décou- 


\title{
Bernard Hue
}

vrant dans la foi l'unique ressource capable de le sauver, et s'y accrochant comme à une bouée de sauvetage. Héroïquement, il s'y est cramponné toute sa vie, criant avec violence qu'il avait raison. Avec la violence dont lui-même, Cuny, n'a jamais pu se départir et grâce à laquelle il s'est trouvé d'emblée en communion avec le poète surnommé le Gorille Catholique ${ }^{16}$. Il fut reconnu par ce gorille, accueilli, certes, comme interprète hors pair, mais aussi comme confident intime, témoin d'exception, qui dira son étonnement d'avoir été l'élu de cet auteur réputé d'accès difficile, dont l'habitant intérieur était tellement ressemblant à lui-même qu'il se plaira à reconnaître :

\begin{abstract}
J'avais bien esquissé pour moi-même un rapprochement entre Claudel et moi, entre moi et certains de ses personnages, certes, mais aussi avec Claudel lui-même, à l'occasion des visites où je l'accompagnais chez la vraie Ysé17.
\end{abstract}

En soulignant un rapprochement a priori non évident entre lui et Claudel, insistant sur la nécessité de ne pas s'en tenir au seul rapport entre le théâtre et le comédien, Cuny ne fait-il pas allusion à une réalité sans lien direct avec le théâtre, à une expérience qui, elle aussi, explique la ressemblance assez inattendue qu'il souligne entre lui et l'auteur de Partage de midi? Ce rapport est d'ordre existentiel. Cuny, passionné de beauté et d'absolu, est un angoissé, un solitaire, un humilié, un rescapé condamné dès l'aube de sa vie au désespoir, - comme le fut le jeune Claudel avant sa conversion -, privé dès son adolescence d'une foi religieuse qui lui eût procuré une solide raison de vivre dans une société que tout lui rend exécrable. Il dira que parmi les rôles qu'il aurait pu jouer il y a celui d'Alceste.

Son théâtre à lui a été la vie; et sa vie a été une tragédie dont il savait qu'elle se terminerait d'une manière sanglante (pour reprendre les termes de Claudel). Une des grandes obsessions de Cuny est la mort. Il fut horrifié devant la lente agonie d'Artaud. Il parlait avec une émotion difficilement contenue de la mort de 
Françoise Dolto. Epris de lumière et de beauté, ne concevant en matière artistique que la perfection, ne découvrant paradoxalement de valeur dans l'action humaine qu'en fonction de l'absolu et de l'instant présent érigé en éternité, - « Il faut vivre l'instant. Il y a pas d'autre éternité ${ }^{18}$, " - il souffrait de tout ce qui était limitation, à peu près, faux semblant, artifice. Horrible, abominable, exécrable, scandaleux étaient parmi ses mots les plus récurrents, de ceux qui lui permettaient d'exprimer son dégoût, avec cette violence qui frappa tant Charles Dullin le jour où, par hasard, l'inconnu Alain Cuny s'était trouvé malgré lui en présence du célèbre professeur d'art dramatique.

\section{Expulsion}

Une violence qui lui venait de loin et non d'une école (il ne fera pas d'études supérieures; malgré son goût pour l'écriture, il hésitera toujours à se consacrer à la littérature). C'est la violence qu'il a découverte, tout enfant, au sein même de sa famille, faisant ainsi penser à Claudel qui évoquera un climat familial non exempt de chicanes entre parents et enfants, entre lui et ses sœurs, déplorant un manque de tendresse de la part de sa mère. Mais l'enfant Cuny, que ses demi-frères et sœurs appelaient le bâtard, subit un sort plus cruel qu'il qualifiera, plus tard, de scandale.

Tout petit, je circulais librement dans les ateliers, dans la salle des machines, seul avec la sœur de mon géniteur, Marie-Ange, qui était très bonne pour moi. Un jour, cet imbécile qu'on me faisait appeler parrain, m’a intimé l'ordre de l'appeler Monsieur! Et voilà, par cet ordre j'ai été littéralement banni du foyer paternel ${ }^{19}$.

Dans un tout autre contexte, il verra dans le drame de Violaine une affaire familiale, dans un milieu édifiant, où dominent la foi catholique, le respect de l'ordre et de la tradition, milieu sévè- 


\section{Bernard Hue}

rement jugé par Mara qui, cinglante, considère que c'est moins la lèpre qui a exclu sa sœur que ses propres parents et son fiancé (dans sa diatribe, elle ne nomme pas Pierre de Craon):

C'est elle seule qu'ils aimaient tous ! et voilà son père qui l'abandonne, et sa mère bien doucement qui la conseille, et son fiancé comme il a cru en elle!

Et c'était là tout leur amour ${ }^{20}$. (Th. II, p. 212)

L'un des premiers mots de Tobie adressés à Azarias, dans L'Histoire de Tobie et de Sara, concerne l'abandon. La réponse d'Azarias peut être entendue comme si elle émanait non de l'acteur, mais d'Alain Cuny lui-même :

Et j'entends ce pauvre enfant sans rien dire qui me demande : Où étais-tu? et pourquoi m'avez-vous ainsi abandonné? (Th. II, p. 1289)

Par delà ce drame de l'amour humain massacré, Cuny porte un regard d'une extrême sévérité sur la représentation dramatique de l'amour qu'offre tout le théâtre de Claudel, sur la fonction de la femme, idéalement conçue comme source de bonheur indispensable à la réalisation plénière de l'homme, mais jamais réalisée dans le cadre de la vie terrestre où, au contraire, elle occasionne, à la manière de la lèpre, les pires souffrances. Incapable de dominer sa vindicte lorsqu'il évoque ce thème principal de l'amour chez Claudel, Alain Cuny met son âme à nu, se fait à la fois Tête d'or, Pierre de Craon, Mesa, héros « brisés $^{21}$ » s'il en est. Dire de l'amour claudélien que c'est un échec est trop peu dire, car il est quelque chose de pire, d'une violence dévastatrice : "C'est une boucherie ${ }^{22}$..."

Image souvent avancée lorsqu'il s'agit de la guerre. Mais n'est-ce pas précisément ce que veut suggérer le comédien qui devait créer le rôle de Simon Agnel, dans Tête d'or? La scène, de ce fait, serait le symbole de la terre où chaque individu, face à l'Autre, où l'homme face à la femme, accomplit sa destinée 
souvent réduite à un combat sans merci. A la différence de Claudel, Cuny ne va pas jusqu'à prophétiser une fin glorieuse, convaincu qu'il n'est d'éternité que dans le présent pleinement vécu, ce qui n'est réalisable que pour celui qui a le privilège de ne pas être irrémédiablement un exclu. Si la joie est compatible avec le drame claudélien, pour Cuny le drame est synonyme de tragédie sans issue.

Chassé par l'amant de sa mère, - expression par laquelle il appelle son géniteur qu'on faisait passer pour son parrain -, Cuny voit dans la soumission, dans le silence de sa mère une attitude proche de celle d'Elisabeth, la mère de Violaine. Elle ne put empêcher que le jeune Alain fût éloigné d'elle et placé dans un orphelinat pour enfants pauvres. Rien d'édifiant dans ces deux familles catholiques, où l'enfant fut accueilli comme un intrus, où sa mort fut vainement espérée. Evoquant ces comportements infâmes, celui qui se fit boxeur probablement pour tenter d'exorciser une violence que sa vie entière ne suffirait pas à faire disparaître se sentira toujours un étranger, un paria. Il dresse avec force et simplicité, sans recherche verbale, sans rien de littéraire, le bilan de sa vie :

Je ne reconnais ma place nulle part. [...] Où que je sois, je suis un intrus. [...]

Je ne me sens rien. Etranger, isolé. [...]

Isolé au milieu de tant de gens? Mais, ajoute-t-il, « les gens édifiants me font horreur ${ }^{23}$ ».

Cette dernière réflexion, sans mettre explicitement la religion en cause, aide à comprendre ce qui conduit Cuny à s'identifier à Pierre de Craon. Non qu'il y ait entière analogie entre Pierre l'architecte et lui, qui déplore de n'avoir rien fait, rien construit, " Je n'ai rien à faire valoir. Aucun bagage. Aucun titre », reconnaît-il avec amertume, pensant peut-être aux premiers mots de Tête d'or se qualifiant d'imbécile, d'ignorant. Pierre de Craon travaille un peu en marge de la petite société que constitue la famille de Violaine, dans laquelle s'harmonisent les valeurs médiévales, 


\section{Bernard Hue}

sauvegarde de la famille et de la religion. Seuls, Mara et Pierre de Craon, bien que chrétiens sincères, ne parviennent pas à s'accommoder de la règle organique de ce monde conformiste. L'un et l'autre, en transgressant l'ordre établi, font figure de révoltés, leur violence les entraînant sur le chemin du crime. Mais l'un et l'autre se considèrent comme des victimes. Il n'échappe à personne, et à Cuny moins qu'à quiconque, que Claudel n'a eu de cesse avec Pierre de Craon que le jour où il le fit bénéficier d'un miracle à Jérusalem où il fut guéri de la lèpre. Quant à Mara, c'est son père lui-même qui lui transmet le pardon en même temps que la vérité sur la nature du baiser au lépreux. L'horreur, aux yeux de Cuny, demeure manifestement dans l'acharnement des forces diverses dont Violaine est scandaleusement la victime que rien d'humain.

\section{Le partage vécu par Cuny}

Enfant blessé, profondément traumatisé, rêvant de devenir peintre, Cuny devait involontairement devenir comédien. Et cela, par la grâce d'une femme, une jeune Danoise, solitaire, rencontrée au temps où il était élève des Beaux $\mathrm{Arts}^{24}$. Il résume l'événement en quelques mots. Tutta était venue à Paris pour faire des études d'art dramatique chez Dullin. Cuny, qui vit alors « en salle de garde à l'asile de Maison-Blanche en face de Ville-Evrard » où Françoise Dolto était interne, revoit Tutta de temps en temps et, un jour, celle-ci le quittant pour se rendre à son cours, il décide de l'accompagner; ils ont le temps d'y arriver, Tutta étant toujours en avance.

[...] je suis entré avec elle dans la salle où Dullin donnait son cours au théâtre de l'Atelier. Les autres élèves sont arrivés, j'ai laissé passer l'heure et, quand Dullin a fait son entrée, j'étais toujours là et je n'ai plus osé sortir. A la fin du cours [...] Dullin, son nez crochu presque au ras de la table, m'a dit : « Et toi, tu es nouveau? " Rouge de peur, j'ai acquiescé. Je ne songeais pas du tout à devenir comédien. »! 
On devine la suite : Dullin lui donne une scène à préparer pour la prochaine fois, et quelle scène! Un extrait de la tragédie de John Ford, 'Tis pity she's a Whore, scène de trahison, de violence conjugale.

\begin{abstract}
j'ai engagé innocemment toute mon énergie. [...] J'ai dû être d'une extrême violence, tout en dirigeant cette violence et tout en articulant bien le texte. Et aussitôt après, Dullin m'a demandé mon âge, ce que je faisais actuellement, et il a ajouté : "Tu peux tout espérer. " Jean-Louis Vaudoyer m'a tout de suite proposé de jouer le rôle d'Hippolyte dans Phèdre, à côté de Marie Bell, à la Comédie-Française dont il venait de prendre la direction. [...] ma vie a changé d'un jour à l'autre.
\end{abstract}

Il a vingt ans. Peut-être songe-t-il au mariage. Il décide de s'acquitter de ses obligations militaires, ce qui l'éloigne provisoirement de Tutta. Il se produit alors ce qui arriva à Claudel au temps de Fou-tcheou.

A vingt ans, j'étais attaché par des liens faux mais intenses à une jeune fille qui s'est mariée précipitamment pendant mon service militaire. La nouvelle de ce mariage m'a réduit à néant comme un imbécile. La souffrance des imbéciles est stupéfiante! Le mariage n'a pas été une réussite, loin de là. Il s'agissait bel et bien d'un viol et d'un enlèvement. L'homme est devenu par la suite ministre. [...]. J'ai vécu sous le signe de cette fille pendant dix ans, inconsolable ${ }^{25}$.

Une telle déception le préparait à comprendre le drame de Claudel, à s'identifier à Mesa, à se faire de la femme et de l'amour une idée dans laquelle se concilient mal l'absolu, le désir de perfection et d'éternité, la réalité qu'à vingt ans il refuse de voir sous l'angle de la banalité. Le drame de l'humiliation le pousse au suicide $^{26}$. Il lui faudra beaucoup d'années pour sortir de ce qu'il appelle sa « vie sépulcrale». 


\section{Bernard Hue}

Cependant, Cuny accorde à l'amour humain une place fondamentale et en cela il est en accord avec les héros claudéliens. S'il définit le rôle de l'homme en ces termes : " tenter d'aimer une femme », on remarquera combien le verbe «tenter » implique le risque d'impossibilité, voire d'échec. D'un côté le désir; de l'autre l'insatisfaction menaçante. Le comédien se rencontre ainsi pleinement avec Claudel qui fait de l'amour l'absolu parfait, dominant tout, et - ce en quoi l'idée de Cuny est en harmonie avec celle que le poète charge Mesa d'exprimer sans craindre le sacrilège -, surpassant jusqu'à Dieu lui-même. Sans doute, le dramaturge ne rompt-il que passagèrement avec ce que Cuny appelle le sacré dogmatique $^{27}$. Mais le comédien demeure persuadé que Claudel est définitivement resté Mesa et qu'il s'est agi pour lui d'une situation très difficile, voire intenable, en tout cas héroïque, que l'auteur de Partage de midi n'est parvenu à vaincre qu'à force de volonté et de prières, en portant sa foi à bout de bras, comme un bouclier. Vision non orthodoxe du grand poète catholique? " Moi seul... ", dit Cuny qui, lui aussi, semblable à Mesa, à Don Camille ou au Don Rodrigue de la deuxième Journée du Soulier de satin, a connu le partage, l'humiliation, l'impossibilité de pardonner, l'angoisse, la colère, la passion crucifiante, " ce terrible amour qu'il faut nous arracher du cœur ${ }^{28}$ ». "Pas une heure, - confie-t-il, octogénaire -, où je n'éprouve pas l'angoisse née dès mon enfance ${ }^{29}$ \%.

Si Alain Cuny reconnaît avec humilité qu'en tant qu'acteur il n'a été qu'un passeur, il clame sa filiation avec l'écrivain qu'il a servi avec la conviction de quelqu'un qui se sent appelé :

Son encre, c'est mon sang. [...] si Claudel m'a profondément transformé, si sa fréquentation et la fréquentation de son œuvre ont soutenu, sans fléchir, ma vie même, en un combat fraternel, il est toujours demeuré une part énigmatique pour moi ${ }^{30}$.

Cette part correspond à ce que le comédien appelle " le noyau d'obscurité », à propos duquel il ajoute : 


\section{Alain Cuny, personnage claudélien}

Qui le comprend? On peut dire : "idéal interprète ", et j'ai eu cette chance, parfois, d'être reconnu comme quelqu'un digne de ce verbe. Mais il s'agissait peut-être d'un malentendu : ce que j'ai toujours voulu faire entendre, par Pierre de Craon, Simon Agnel, et Couvre bien sûr, plus encore peut-être, c'est ce qui résiste. Ce que rien ne peut dissoudre.

Seul peut-être, Alain Cuny, comme il le pensait, sera parvenu jusqu'à ce point extrême, jusqu'au niveau du noyau où le rideau de ténèbres interdit à quiconque de pénétrer, mais d'où se sont échappés les personnages multiformes, remodelés par l'imagination du poète et scéniquement portés pleinement à la vie par le comédien qui, avec le plus grand naturel, se sentait chacun d'eux.

Oui, j'ai désiré jouer le rôle d'Alceste et je ne l'ai jamais fait. Lui et certains personnages de Claudel, ce sont des personnages auxquels je ressemble tellement que je veux de toutes mes forces leur échapper. Ils sont là pour que je ne sois pas pareil à eux. [...] Ce n'est pas la péripétie qui me rend malade, elle ne fait que réactiver la boule de maladie qui est en $\mathrm{moi}^{31}$.

Ne serait-ce pas à cette boule si semblable à la sienne que renvoie Cuny quand il parle d'un noyau d'obscurité chez Claudel? Une obscurité devant laquelle le tragédien s'est incliné, sûr d'avoir été de ceux qui auront eu le rare privilège, par delà l'admiration pour le poète, de découvrir en ce dernier leur propre miroir.

\section{Notes}

1 A. Cuny, Le Désir de parole, Conversations et rencontres avec Alfred Simon, Ed. la manufacture, Lyon, 1989, p. 157. La référence à ces entretiens sera désormais appelée par Désir. 


\section{Bernard Hue}

2 Cuny fut très frappé par son horoscope du 18 juin 1937 établi par Artaud. Celui-ci en tirait la conclusion suivante : «Vous êtes divisé entre la chasteté et le désir. » Désir, p. 53. Cuny vivait alors son propre « partage » de midi.

3 Claudel, Théâtre, Pléiade, 1965, t. II, p. 1403, Préface pour la thèse inédite de Jean Tipy "Les différentes versions de L'Annonce faite à Marie », 29 juin 1954. Quelques années plus tôt, Claudel déclarait, à propos d'Anne Vercors qui, aux yeux de Jean Amrouche, semblait effacer Tête d'or : "C'est possible, bien que jamais je ne puisse m'identifier avec un seul de mes personnages en particulier. » Mémoires improvisés, Gallimard, 1969, p. 268.

4 Ibid., p. 1403.

5 Lettre du 15 juillet 1942 à Françoise de Marcilly, Lettres à une amie, Bayard, 2002, p. 273. - On sait suite à quel hasard Cuny, très jeune, se trouva malgré lui mis en face de Dullin, et comment, sans avoir songé au théâtre, sans avoir suivi aucun cours d'art dramatique, il fut d'emblée « reconnu » comme un authentique comédien, immédiatement recommandé à la Comédie Française. En 1942, Claudel parlant du projet de Jean-Louis Barrault concernant Le Soulier de satin, ajoute qu'on s'occupe aussi « de monter L'Echange pour décembre, avec l'aide de Ledoux et d'un garçon très doué appelé Alain Cuny. » C'est, semble-t-il, la première mention du nom de Cuny sous la plume de Claudel qui dira toujours le plus grand bien de cet acteur qu'il estima à l'égal de J.-L. Barrault lui-même. En 1948, quelques mois après avoir qualifié Cuny d'admirable acteur en puissance (Journal, Pléiade, 1969, t. II, p. 617), Claudel n'hésite pas à noter à propos du Soulier de satin: "Je dis que nous pouvons nous passer parfaitement de Jean-Louis Barrault. En dehors de lui nous avons toute l'interprétation primitive, je voudrais Alain Cuny à sa place. » (Ibid, p. 631). La même année, obligé de renoncer au rôle de Jacques Hury qu'il lui destinait, Claudel écrit à Alain Cuny à propos du refus d' Hébertot: " C'est un énorme crève-cœur pour moi, car vous étiez pour moi le personnage idéal. Je suis désemparé! »(Désir, hors texte).

6 La Comédie-Française songe alors à jouer L'Annonce faite à Marie; son projet n'aboutira qu'en 1955.

7 Des projets sont restés sans lendemain. Alain Cuny devait jouer dans L'Echange, en 1942, avec Fernand Ledoux, mais cette pièce fut interdite par la censure. Il a également été pressenti pour le rôle de Don Camille dans Le Soulier de satin, créé en 1943, Claudel tenant beaucoup à lui pour cet emploi; ce projet n'aboutit pas. Le poète avait insisté pour que Cuny fût Jacques Hury, dans L'Annonce..., en 1948 


\section{Alain Cuny, personnage claudélien}

(voir lettre de Barrault, in Désir, hors texte), et Cuny lui-même n'a jamais caché qu'il aurait aimé jouer le rôle de Mesa, regrettant, sans trop d'amertume apparente, de ne pas avoir été sollicité. Suite au triomphe parisien de L'Annonce... et dans la perspective de l'Année Sainte (1950), Hébertot avait préparé la représentation du drame mystique qui devait avoir lieu dans une salle de la basilique SaintPierre. Cuny aurait vraisemblablement fait partie de la distribution envisagée. Pie XII s'était montré favorable à cette représentation. Mais le Sacré Collège interdit cette représentation. (voir BSPC $\left.\mathrm{n}^{\circ} 138,1995\right)$. Cuny trouva quelque compensation à ces occasions manquées dans les lectures publiques qu'il fit jusqu'à la fin de sa vie. Il ne cachait pas la satisfaction qu'il tirait de sa lecture intégrale à une voix de Partage de midi, enregistrée sur disque (ORTF, 16 tours, O.P.E.R.A. 302).

$8 \mathrm{La}$ Comédie-Française songe alors à jouer L'Annonce faite à Marie; son projet n'aboutira qu'en 1955.

9 Saint-Malo, le 27 mars 1983. A. Cuny a participé à l'émission de France-Culture, Les Rameaux, Le Chemin de la Croix, La Nuit de Pâques (11 avril 1968). La même année, il est le récitant dans le disque Le Chemin de la Croix, musique de Dom Clément Jacob, ensemble instrumental sous la direction du R.P. Picard. DNO 531.

10 Contrairement à ce qu'on pourrait croire, ce n'est pas Cuny qui a pensé le premier à transposer cinématographiquement la pièce de Claudel. Il le souligne lui-même, voir Désir, p. 131. S'il a accepté la proposition qui lui a été faite, c'est, dit-il, « à cause de Claudel. " Dès février 1968, son scénario est prêt, voir $\mathrm{BSPC} \mathrm{n}^{\circ} 30$, p. 29. Si la réalisation du film ne sera définitive qu'en 1991, c'est en raison de difficultés, en particulier financières, qui faillirent faire échouer le projet. Cuny déplorait l'acharnement qu'il voyait, à tort ou à raison, entretenir contre lui. Ce film a reçu un très bon accueil. Il reste, malheureusement, peu connu à cause d'une distribution qui échappe aux règles de la grande commercialisation cinématographique. Cuny en parle avec humour; dans un télégramme envoyé à un ami pour l'inviter à assister à la projection de ce film, il écrit : « Le film est chez les épiciers, il ne m'appartient plus, [...] "Bernard Fabre, Une amitié. A la mémoire d'Alain Cuny, Edition Séguier, p. 39. Le film de Cuny a reçu le prix Georges Sadoul.

11 Bien qu'il eût songé à Isabelle Adjani pour le rôle de Violaine (après quelques essais, l'idée fut abandonnée), A. Cuny décida de se passer d'acteurs professionnels (auxquels il refusait le titre de stars). Ne se considérant pas comme tel, il s'attribua le rôle d'Anne Vercors 
12 Parmi les nombreux comédiens et metteurs en scène auxquels Claudel a eu affaire, rares sont ceux dont il n'a eu qu'à se féliciter. Cuny apparaît comme une exception. De plus, les deux hommes, dont la différence d'âge était grande (Cuny étant né en 1908), entretinrent une amitié qui fit de ce dernier l'un des rares témoins, et peut-être le seul, des tête à tête entre les vrais personnages de Partage de midi, Mesa et Ysé, lors d'épisodiques et brèves retrouvailles à la fin de leur vie.

13 Désir, p. 114. De son côté, Claudel, recourant à l'autodérision, rappelle, à la fin de sa vie, que les personnages qu'il a créés sont déplaisants, et, précisant sa pensée, il ajoute : aussi « claudéliens qu'ils peuvent l'être. » Mémoires improvisés, p. 278.

14 Ibid., p. 160. Il ajoute que s'il n'a pas joué Mesa, il a fait un enregistrement du Partage de midi pour les « Lectures à une voix. » de Michel Polac. Disque paru en 1966, dans la Collection Paul Claudel, 16 tours, ORTF, O.P.E.R.A. 302.

15 Désir, p. 181 et p. 153. «On commence à savoir que Claudel est un rescapé. » Ibid. p. 187 : « Je pense d'ailleurs avoir joué un rôle dans cette découverte. »

16 Désir, p. 170. Le jugement du comédien n'a rien d'irrévérencieux à l'égard du poète qui se taxe lui-même de prêtre manqué : «Un prêtre manqué! quelle tristesse! " Lettre du $1^{\text {er }}$ août 1937, in Lettres à une amie. Correspondance avec Françoise de Marcilly, Bayard, 2, p. 144.

17 J. I, p. 999 et II, p 623.

18 Désir, p. 120. Dans un de nos entretiens, A. Cuny m'a confié que Claudel, à Paris, faisait appel à lui de temps à autre, lui demandant de venir le prendre pour le conduire à l'adresse où résidait alors Rosalie Vetch. Le poète tenait à ce que Cuny demeurât présent à ses côtés durant ces visites à « sa vieille maîtresse ", ce qui ne laissait pas de l'intriguer.

19 Ibid., p. 115.

20 Ibid., p. 124.

21 Le texte de la $1^{\text {ère }}$ version a une formulation moins cinglante; Mara s'adressant à son père et à son mari utilise le pronom de la deuxième personne : Vous l'aimiez tous! Dans la version définitive, la troisième personne exprime un mépris né de la haine et du désespoir.

22 Désir, p. 177. Commentant quelques vers de Tête d'or, Cuny y voit une transcription pure et simple de la souffrance de Claudel, « c'est la sienne, la sienne seule, [...]. C'est un Titan constamment réduit en lambeaux, qui se redresse, qui veut la belle pomme rouge et qui sait 
qu'il n'est qu'un cul-de-jatte, comme Rodrigue, comme Tête d'or, comme Mesa. Claudel les représente tous physiquement brisés. »

23 Ibid., p. 128. Une observation très explicite quant au point de vue de Claudel sur l'amour tel qu'il l'a peint dans Partage de midi se rencontre dans son Journal, en juin 1948. Il s'agit d'une citation empruntée à Maupassant et consacrée à l'impossible rencontre réelle de deux êtres qui s'aiment. "Jamais rien ne pourra briser cette invisible frontière qui met les êtres dans la vie aussi loin l'un de l'autre que les étoiles du ciel. » J. II, p. 643.

24 Désir, pp. 149, 155, 145.

25 Cuny fait le récit de cet épisode dans Désir, p. 100.

26 Désir, p. 185. Cuny se mariera en 1962, âgé de 54 ans; il divorcera sept ans plus tard. Père d'un fils qu'il a peu connu, il dira son regret de n'avoir pas joué le rôle qui était le sien. On pense à la réflexion de Michelle Manceaux qui avait commencé à recueillir les mémoires du comédien : "J'avais dû deviner chez cet acteur à la présence rare, chez cet homme traversé d'éclairs et frappé de foudre, à la fois arbre et bûcheron, la conduite d'échec qui le pousse à détruire ce qu'il entreprend. » Grand reportage, Edition du Seuil, 1980, p. 91. Exigeant, sévère dans ses jugements, violent parfois dans ses réactions, A. Cuny se savait taxé d'ingratitude, de manquer de camaraderie, à quoi il répliquait avec une superbe assurance et une ironie qui en dit long sur son sentiment religieux : « comme si nous étions faits pour nous aimer les uns les autres! Si c'était le cas, pourquoi Dieu a-t-il été contraint d'envoyer son fils au massacre pour nous le rappeler? » Désir, p. 138.

27 « J'avais pris des barbituriques. On m'a tiré de là avec des pincettes, à un demi cheveu près [...]" Désir, p. 172. Il rappelle, avec amertume, ce que cette mort programmée avait inspiré à l'une de ses demi-sœurs : «Tu n'as donc pas pensé à ce qu'on allait dire de nous? » Voilà les cloisons qui m'ont emprisonné toute ma vie. »

28 Désir, p. 143. Type de sacré que Cuny qualifie de «produit de confiserie ».

29 Claudel, Le Chemin de la Croix, Euvre poétique, Pléiade, 1957, p. 490.

30 Désir, p. 179. « La tradition chrétienne, écrit Bernard Fabre, lui offrait une raison de veiller sur le mal qui le rongeait. [...] Il savait pourtant que le sacrifice, de soi ou d'autrui, loin de porter remède au mal, l'excite en activant l'angoisse. Il trouvait terrifiante la fable de la mère qui accepte de donner la vie à un fils destiné au supplice de la croix. 


\section{Bernard Hue}

«Quelle horreur! » Mais il reconnaissait sa propre histoire dans cette fable. "Une amitié. A la mémoire d'Alain Cuny, Edition Séguier, p. 16.

31 Cité par Armelle Héliot in « Alain Cuny et Paul Claudel. L'entretien infini », Bulletin de la Société Paul Claudel, n 136, 1994, p. 3.

32 Ibid., p. 160. Daniel Gélin voyait en Cuny « un grand anarchiste mystique, un médiéval, quelqu'un de formidable » (in Ouest France, mai 1994). 